\title{
Comparisons of Water-Equivalent Diameter Measured on Images of Abdominal Routine Computed Tomography with and without A Contrast Agent
}

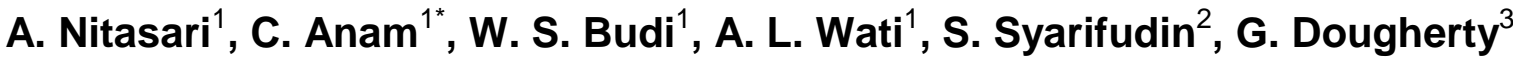 \\ ${ }^{I}$ Department of Physics, Faculty of Sciences and Mathematics, Diponegoro University, Jl. Prof. Soedarto SH, \\ Tembalang, Semarang 50275, Indonesia \\ ${ }^{2}$ Department of Radiology, Dr. Kariadi Hospital, Jl. Dr. Sutomo No.19, Semarang Selatan, Semarang 50244, Indonesia \\ ${ }^{3}$ Department of Applied Physics and Medical Imaging, California State University Channel Islands, Camarillo, CA, USA
}

\section{ARTICLE INFO}

Article history:

Received 27 December 2020

Received in revised form 02 March 2021

Accepted 08 March 2021

Keywords:

Abdominal routine

Size-specific dose estimate (SSDE)

Water-equivalent diameter $\left(\mathrm{D}_{\mathrm{W}}\right)$

\begin{abstract}
A B S T R A C T
The size-specific dose estimate (SSDE) is a metric for an estimation of patient dose in computed tomography (CT). The SSDE strongly depends on the water-equivalent diameter $\left(\mathrm{D}_{\mathrm{W}}\right)$. In abdominal $\mathrm{CT}$ examinations, a contrast agent is sometimes used to more clearly visualize tissue lesions. The Hounsfield unit (HU) of CT images with and without the use of a contrast agent at specific areas is slightly different and it may affect the $\mathrm{D}_{\mathrm{W}}$ value. This study aimed to compare the $\mathrm{D}_{\mathrm{W}}$ values calculated from axial CT images in patients who had undergone routine abdominal scans both with and without the use of a contrast agent. Axial images of 144 patients with a weight range of $3.5 \mathrm{~kg}$ to $90 \mathrm{~kg}$ who had undergone routine abdominal scans both with and without the use of a contrast agent using a Siemens Sensation 64 CT scanner were retrospectively collected. The $\mathrm{D}_{\mathrm{W}}$ values were automatically calculated using the Matlab-based IndoseCT (version 15a) software. The results show the percentage difference between $\mathrm{D}_{\mathrm{W}, \text { contrast }}$ and $\mathrm{D}_{\mathrm{W} \text {,non-contrast }}$ is below $2 \%$. As a result, the mean $\mathrm{SSDE}_{\text {contrast }}$ is $1.5 \%$ smaller than $\mathrm{SSDE}_{\text {non-contrast }}$. Due to the effect of a contrast agent on the $\mathrm{D}_{\mathrm{W}}$ and SSDE values is below $2 \%$, the axial images of CT abdomen without the use of a contrast agent can be used as the accurate estimation of $\mathrm{D}_{\mathrm{W}}$ and SSDE for images with the use of a contrast agent.
\end{abstract}

(C) 2021 Atom Indonesia. All rights reserved

\section{INTRODUCTION}

Computed tomography (CT) was introduced in the 1970s and has had a crucial role in the diagnostic field due to its excellent image quality $[1,2]$. Image acquisition in diagnostic CT is very fast due to the advances in acquisition techniques such as helical and multi-slice CT (MSCT). These advantages lead to the growing use of CT in numerous applications. However, its disadvantage is that it contributes to the highest radiation exposure in the medical field [3-5]. Hence, its implementation must be carefully and prudently optimized so that its advantages outweigh its disadvantages.

Estimating accurate patient dose is important. Accurate estimation of patient dose relies on the

\footnotetext{
*Corresponding author.

E-mail address: anam@fisika.fsm.undip.ac.id

DOI: https://doi.org/10.17146/aij.2021.1112
}

metric known as the size-specific dose estimate (SSDE) [4-6]. Since SSDE was announced, several softwares have been developed to measure the effective dose and organ dosage based on SSDE $[9,10]$. Several studies reported that SSDE was used to represent dose optimization values such as diagnostic reference level (DRL) and acceptable quality dose (AQD) [11-14]. SSDE is estimated from the output dose or the volume CT dose index $\left(\mathrm{CTDI}_{\mathrm{vol}}\right)$ and patient characteristics. In this case, the patient characteristics include X-ray attenuation and patient size. The X-ray attenuation is based on many factors, such as type of material and its density and energy of X-ray beam. The X-ray attenuation is a fundamental parameter effecting X-ray absorption and the dose absorbed by patient. The patient characteristics are generally represented by the water-equivalent diameter $\left(D_{W}\right)[15,16]$ of the patient. Its value can be determined from patient images $[7,17,18]$. 
$\mathrm{X}$-ray attenuation in $\mathrm{CT}$ is expressed in Hounsfield units (HU). Each body tissue has different HU values; however, the resulting contrast for distinguishing adjacent tissue may be relatively small. Thus, a contrast agent is needed to improve the image contrast of the desired body tissue $[19,20]$. In a CT examination, the contrast agent is iodine-based [21,22]. Common indications for the use of a contrast agents on CT abdominal scans include acute appendicitis, cancer staging, diverticulitis, and pancreatitis [23].

Because the pixel values (HU) within image change in some tissues before and after agent contrast injection, the $\mathrm{D}_{\mathrm{W}}$ and the SSDE may change as a result. Based on our best knowledge, no previous study investigated the $\mathrm{D}_{\mathrm{W}}$ and SSDE before and after contrast agent injection. As consequence, the $\mathrm{D}_{\mathrm{W}}$ and SSDE before and after contrast agent injection are considered the same without justification from the careful study. Therefore, this study aimed to calculate the $D_{\mathrm{W}}$ values based on axial images of CT examinations with and without the use of a contrast agent. In addition, we also evaluated the SSDE value before and after contrast agent injection. The results of this study will be useful to confidently consider the $\mathrm{D}_{\mathrm{W}}$ and SSDE for CT examinations with a contrast agent administration.

\section{MATERIALS AND METHODS}

\section{Patient images}

We calculated the $D_{\mathrm{w}}$ values of 144 patients with a weight range from $3.5 \mathrm{~kg}$ to $90 \mathrm{~kg}$ who underwent routine abdominal CTs both with the use of a contrast agent referring to the portal venous phase and without the use of a contrast agent. The contrast agent was Iopamidol 370 (IOP 370). The patients were scanned using the Siemens Sensation 64 CT scanner installed at Dr. Kariadi Hospital, Semarang, Central Java, Indonesia. The routine abdominal protocol for pediatric patients, with and without the use of a contrast agent, was $120 \mathrm{kVp}, 85 \mathrm{~mA}, 1.4$ pitch factor, and $28.8 \mathrm{~mm}$ total collimation width. For adult patients the tube current was increased to $200 \mathrm{~mA}$, with the same of all other settings. All the exposure parameters mentioned were obtained from each patient's DICOM header. In addition, both the abdominal CT examination protocol in children and adults applied the tube current modulation (TCM) technique. The TCM was to manage tube loading to optimize dose delivery. Once the TCM was used, the tube loading for each slide would be different due to different patient equivalent thickness. Meanwhile, the CTDI ${ }_{\mathrm{vol}}$ were obtained from the CT dose summary as shown in Fig. 1.

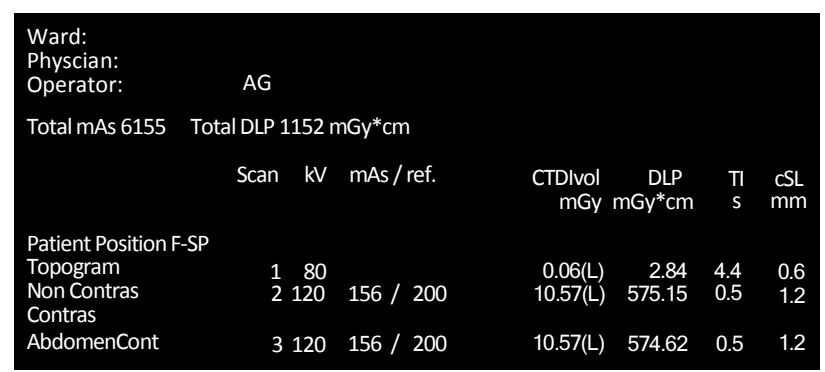

Fig. 1. Captured CT dose summary from Siemens Sensation 64 CT scanner.

\section{Calculation of $D_{w}$}

The $D_{W}$ of a patient can be calculated from the mean $\mathrm{HU}$ in the region of interest (ROI). $\mathrm{D}_{\mathrm{W}}$ was calculated using Eq. (1).

$$
D_{w}=2 \sqrt{\left[\frac{1}{1000} \overline{H U}+1\right]} \frac{A}{\pi}
$$

where $A$ is the area of the patient and $\overline{H U}$ is the mean HU value within the patient [24]. In this study, we used axial image data extracted from each patient's DICOM files to calculate the $D_{w}$ value. The $D_{w}$ was automatically calculated using Matlab-based IndoseCT version 15 a software $[18,25]$. The IndoseCT automatically made the contouring as shown in Fig. 2 and calculated the $\mathrm{D}_{\mathrm{W}}$ according to the selected tool. For the $\mathrm{D}_{\mathrm{w}}$ calculation on the IndoseCT, we used the "3D" tool and selected a slice number of 9 (in Fig. 3), i.e., 9 slices were selected from the axial CT images to represent the water-equivalent diameter along the longitudinal axis. A previous study [6] found that $D_{W}$ values calculated using these settings had a mean percentage difference value less than $1 \%$ compared to the $D_{\mathrm{W}}$ from all slices [6]. $\mathrm{D}_{\mathrm{W}}$ value calculations for each patient were performed using axial CT images with and without the use of a contrast agent.

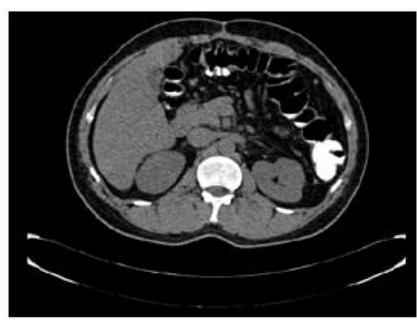

(a)

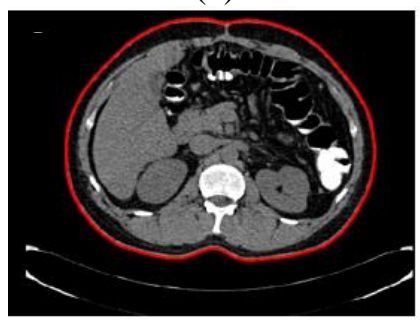

(b)

Fig. 2. The auto-contouring by the IndoseCT. (a) original image and (b) auto-contouring result. 


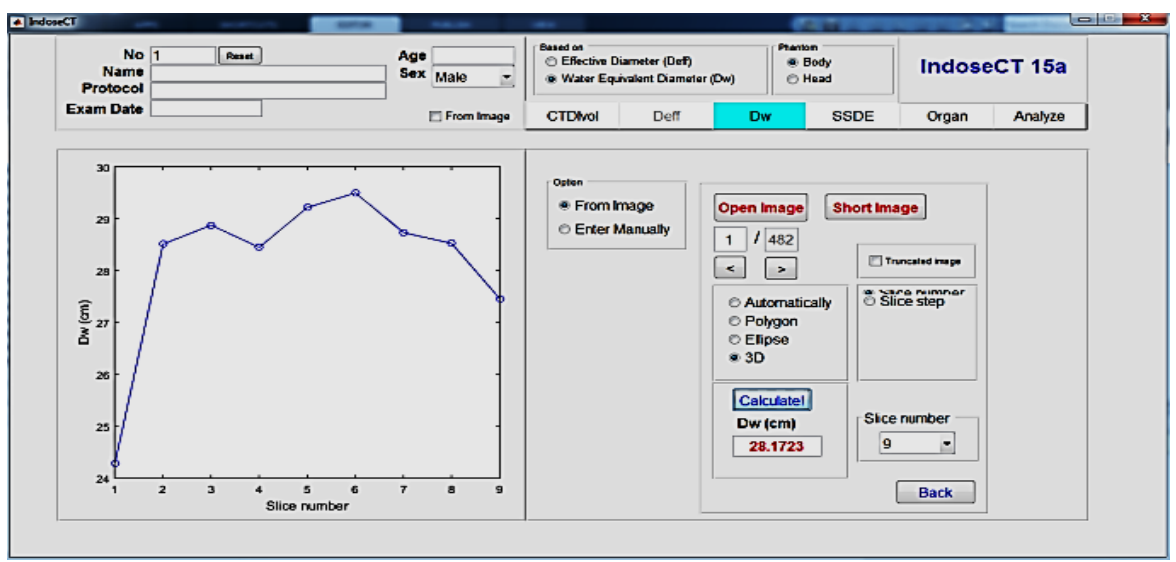

Fig. 3. The $D_{w}$ calculation using $3 \mathrm{D}$ tool and a slice number of 9 using the IndoseCT.

\section{Calculation of SSDE}

SSDE values can be calculated by $\mathrm{CTDI}_{\mathrm{vol}}$ which can be obtained from a CT scanner dose summary [18] and the size-conversion factor (f) using Eq. (2).

$$
S S D E=C T D I_{v o l} \times f
$$

The size-conversion factor of $\mathrm{D}_{\mathrm{W}}$ can be obtained from the AAPM report 220 [7]. In this study, both SSDE values $\left(\mathrm{SSDE}_{\text {contrast }}\right.$ and $\mathrm{SSDE}_{\text {non-contrast }}$ ) were automatically calculated using the IndoseCT version 15a [18].

\section{Relationship between $D_{\mathrm{w}, \text { contrast }}$ and $\mathrm{D}_{\mathrm{W} \text {,non-contrast }}$}

The number of patients for the $\mathrm{D}_{\mathrm{w}}$ calculation using axial CT images with the use of a contrast agent $\left(D_{W, c o n t r a s t}\right)$ referring to the portal venous phase and without the use of a contrast agent ( $\mathrm{D}_{\mathrm{W} \text {,non-contrast }}$ ) was 144 . The $\mathrm{D}_{\mathrm{W} \text {,contrast }}$ and the $\mathrm{D}_{\mathrm{W}, \text { non-contrast }}$ values for abdominal scans were compared. The percentage difference between $\mathrm{D}_{\mathrm{W} \text {,contrast }}$ and $\mathrm{D}_{\mathrm{W} \text {,non-contrast }}$ was calculated using Eq. (3).

$$
P D=\left(\frac{D_{W, \text { contrast }}-D_{W, \text { non-contrast }}}{D_{W, \text { non-contrast }}}\right) \times 100
$$

\section{RESULTS AND DISCUSSION}

Figure 4 presents the axial CT images of the abdomen with and without a contrast agent. It shows that the axial CT images of the abdomen with the use of a contrast agent provides better visualization of the portal vein and solid organs, i.e., the liver, spleen, kidney, and pancreas than that without the use of a contrast agent due to the HU values increase in these areas [27,28]. The mean HU value in some areas of the axial CT image of the abdomen was $22.6 \%$ greater than that without the use of a contrast agent.

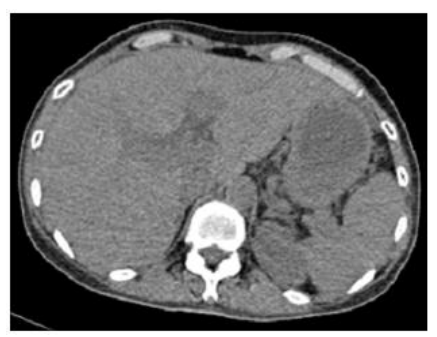

(a)

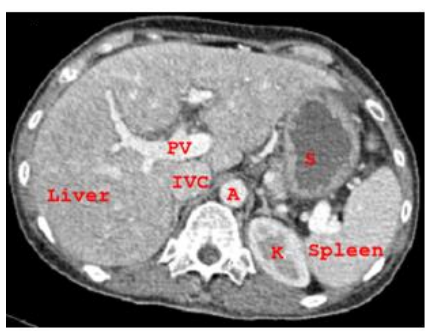

(b)

Fig. 4. (a) The axial CT image of the abdomen without the use of a contrast agents, and (b) The axial CT image

of the abdomen with the use of a contrast agents showing the liver; PV, portal vein; IVC, Inferior vena cava;

A, aorta; S, stomach; P, pancreas; K, kidney, and spleen.

\section{Relationship between $D_{\mathrm{W}, \text { contrast }}$ and $D_{\mathrm{W} \text {,non-contrast }}$}

The relationship between $\mathrm{D}_{\mathrm{W} \text {,contrast }}$ and $\mathrm{D}_{\mathrm{W}, \text { non-contrast }}$ is shown in Fig. 5a. The correlation is strong $\left(\mathrm{R}^{2}=0.974\right)$. Based on this finding, the $\mathrm{D}_{\mathrm{W}, \text { contrast }}$ can be accurately calculated from $\mathrm{D}_{\mathrm{W} \text {,non-contrast. }}$ Figure $5 b$ shows the box-plot diagrams of $D_{W, \text { contrast }}$

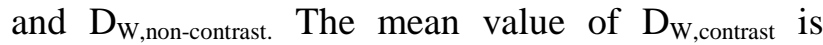
$1.2 \%$ greater than the mean value of $\mathrm{D}_{\mathrm{W}, \text { non-contrast }}$. This indicates that there is only a small difference of a contrast agent on the $D_{W}$ value because the use of a contrast agent leads to an increase the $\mathrm{HU}$ values only in some organs $[19,20,26]$. This finding is consistent with the very recent study [29]. They reported that $D_{W}$ after contrast agent injection is only $0.21 \mathrm{~cm}$ greater than $\mathrm{D}_{\mathrm{W}}$ before contrast agent injection. 


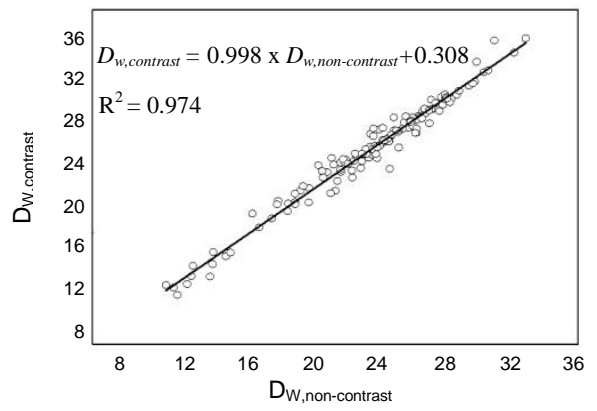

(a)

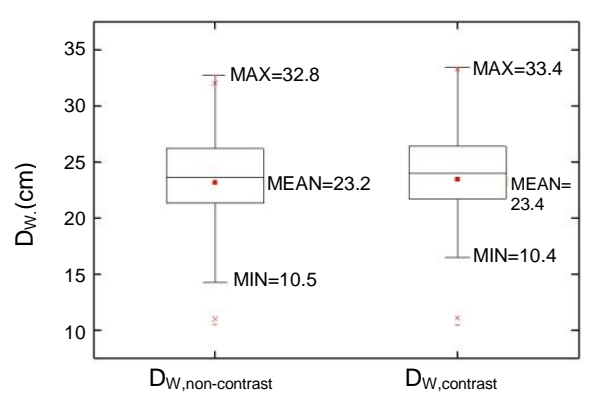

(b)

Fig. 5. (a) Relationship between $D_{W, \text { contrast }}$ and $D_{W, \text { non-contrast; }}$; and (b) Box-plots for $\mathrm{D}_{\mathrm{W}, \text { contrast }}$ and $\mathrm{D}_{\mathrm{W} \text {,non-contrast. }}$

\section{Relationship between $\mathrm{CTDI}_{\mathrm{vol}, \text { contrast }}$ and CTDI $_{\text {vol,non-contrast }}$}

The relationship between $\mathrm{CTDI}_{\mathrm{vol}, \text {,ontrast }}$ and $\mathrm{CTDI}_{\text {vol,non-contrast }}$ is shown in Fig. 6a. The correlation is very strong $\left(\mathrm{R}^{2}=0.995\right)$. Box-plots in Fig. 6b show a very small difference $(0.1 \%)$ with the mean value of $\mathrm{CTDI}_{\mathrm{vol}, \text { contrast }}$ greater than the mean value of $\mathrm{CTDI}_{\mathrm{vol}, \text { non-contrast. This difference is not }}$ significant. This is because the same protocol scan is used for routine abdominal scans with and without the use of a contrast agent. For information, the CT scanner used at our institution applies the TCM technique. In the TCM technique, the $\mathrm{CTDI}_{\mathrm{vol}}$ is affected by the tube loading and it is affected by the size of the patient. The correlation between tube loading and $\mathrm{CTDI}_{\mathrm{vol}}$ has been reported by the previous study [18].

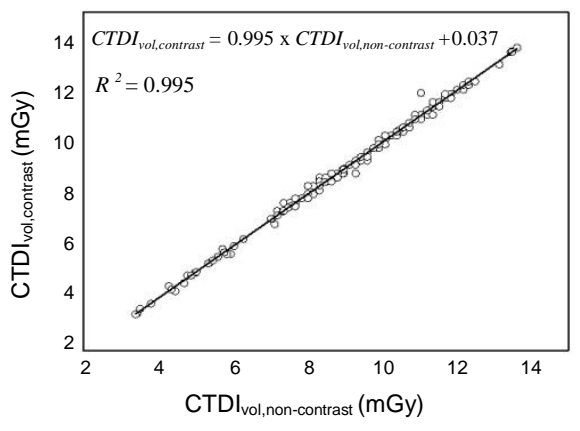

(a)

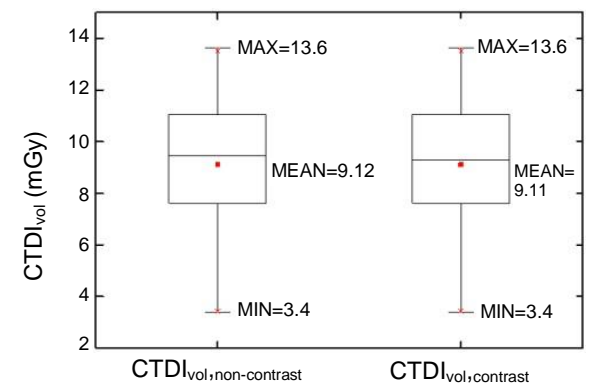

(b)

Fig. 6. (a) Relationship between $\mathrm{CTDI}_{\mathrm{vol}, \text { contrast }}$ and CTDI $_{\text {vol,non-contrast }}$, and (b) Box-plots for $\mathrm{CTDI}_{\mathrm{vol}, \text { contrast }}$ and $\mathrm{CTDI}_{\text {vol,non-contrast. }}$

\section{Relationship between SSDE $_{\text {contrast }}$ and SSDE $_{\text {non-contrast }}$}

The relationship between $\mathrm{SSDE}_{\text {contrast }}$ and $\mathrm{SSDE}_{\text {non-contrast }}$ is shown in Fig. 7a. The correlation is strong $\left(\mathrm{R}^{2}=0.943\right)$. The box-plots (Fig. $7 \mathrm{~b}$ ) show that the mean value of $\mathrm{SSDE}_{\text {contrast }}$ is $1.5 \%$ smaller than the mean value of $\operatorname{SSDE}_{\text {non-contrast }}$, which is due to $\mathrm{D}_{\mathrm{W} \text {,contrast }}$ being $1.2 \%$ greater than the mean value

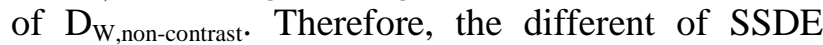
before and after contrast agent is not significant. This result is consistent with the very recent study which reported that SSDE from images with contrast agent is rougly $1 \%$ lower than those from images without contrast agent [29]. AAPM reported that if the difference between the pre- and post-scan values of $\mathrm{D}_{\mathrm{W}}$ and SSDE is below $10 \%$, the final values used may be taken from the pre-scan values [7].

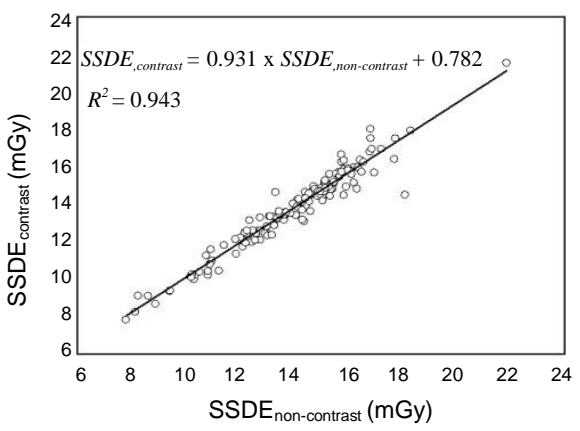

(a)

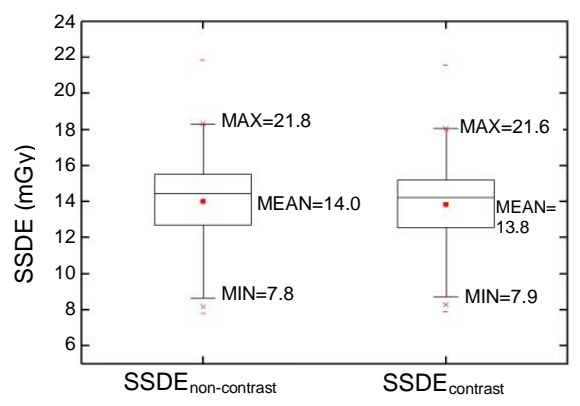

(b)

Fig. 7. (a) Relationship between $\mathrm{SSDE}_{\text {contrast }}$ and SSDE $_{\text {non-contrast }}$, and (b) Box-plots for SSDE contrast and $\mathrm{SSDE}_{\text {non-contrast. }}$ 


\section{CONCLUSION}

As expected, $\mathrm{D}_{\mathrm{W}, \text { contrast }}$ is greater than $\mathrm{D}_{\mathrm{W}, \text { non- }}$ contrast. The percentage difference between $\mathrm{D}_{\mathrm{W}, \text { contrast }}$ and $\mathrm{D}_{\mathrm{W} \text {,non-contrast }}$ is $1.2 \%$. As a result, the mean $\mathrm{SSDE}_{\text {contrast }}$ is $1.5 \%$ smaller than $\mathrm{SSDE}_{\text {non-contrast. }}$ Due to the effect of a contrast agent injection on the $D_{\mathrm{W}}$ and SSDE values is not significant (below $10 \%$ ), the axial images of CT abdomen without the use of a contrast agent can be used to estiamate the $\mathrm{D}_{\mathrm{w}}$ and SSDE from images with the use of a contrast agent injection.

\section{ACKNOWLEDGEMENT}

This work was funded by the World Class Research University (WCRU), Diponegoro University, No. 118-08/UN7.6.1/PP/2021.

\section{AUTHOR CONTRIBUTION}

A. Nitasari and C. Anam equally contributed as the main contributors of this paper. All authors read and approved the final version of the paper.

\section{REFERENCES}

1. G. D. Rubin, Radiol. 273 (2014) S45.

2. Anonymous, Sources and Effects of Ionizing Radiation, UNSCEAR 2008 Report to the General Assembly with Scientific Annexes Vol. 1, United Nations Publication, New York (2010) 1.

3. G. Kirova and G. Hadjidekov, Rentgenol. i Radiol. 44 (2005) 166.

4. S. P. Power, F. Moloney, M. Twomey et al., World J. Radiol. 8 (2016) 902.

5. Anonymous, Sources and Effects of Ionizing Radiation, UNSCEAR 2008 Report to the General Assembly with Scientific Annexes Vol. 1, Annex A Medical Radiation Exposures, United Nations Publication, New York (2010) 23.

6. C. Anam, F. Haryanto, R. Widita et al., J. Appl. Clin. Med. Phys. 17 (2016) 320.

7. AAPM, Report No. 220 - Use of Water Equivalent Diameter for Calculating Patient Size and Size-Specific Dose Estimates (SSDE) in CT (2014) 1.
8. R. D. A. Khawaja, S. Singh, B. Vettiyl et al., Am. J. Roentgenol. 204 (2015) 167.

9. P. Sahbaee, W. P Segars and E. Samei, Med. Phys. 41 (2014) 072104.

10. A. Fahmi, C. Anam, Suryono et al., Radiat. Prot. Dosimetry 188 (2020) 522.

11. R. Imai, O. Miyazaki, T. Horiuchi et al., Pediatr. Radiol. 45 (2015) 345.

12. A. Mohammadbeigi, K. Khoshgard, A. Haghparast et al., Radiat. Prot. Dosimetry 186 (2019) 496.

13. A. Mehdipour and M. Parsi, Radiat. Prot. Dosimetry 185 (2019) 176.

14. K. M. Kanal, P. F. Butler, D. Sengupta el al., Radiol. 284 (2017) 120.

15. M. Bostani, K. McMillan, P. Lu el al., Med. Phys. 42 (2015) 958.

16. C. Anam, D. Adhianto, H. Sutanto et al., J. Xray. Sci. Technol. 28 (2020) 695.

17. C. Anam, F. Haryanto, R. Widita et al., J. Phys.: Conf. Ser. 1204 (2019) 012002.

18. C. Anam, F. Haryanto, R. Widita et al., Int. J. Radiat. Res. 16 (2018) 289.

19. E. Amato, I. Salamone, S. Naso et al., Am. J. Roentgenol. 200 (2013) 1288.

20. L. Hrvoje and M. W. Grinstaff, X-Ray Computed Tomography Contrast Agents, ACS Publicatios, Washington D.C. (2014).

21. P. Sahbaee, E. Abadi, W. P. Segars et al., Radiol. 283 (2017) 749.

22. A. Suwannasri, R. Kaewlai and S. Asavaphatiboon, J. Phys.: Conf. Ser. 694 (2016) 012045.

23. J. V. Rawson and A. L. Pelletier, Am. Fam. Physician 88 (2013) 312.

24. C. Anam, I. Arif, F. Haryanto et al., Radiat. Prot. Dosimetry 185 (2019) 34.

25. C. Anam, F. Haryanto, R. Widita et al., J. Phys.: Conf. Ser. 694 (2016) 012030.

26. K. T. Bae, Radiol. 256 (2010) 32.

27. K. Guite, Louis and F. Lee, Sel. Top. Comput. Tomogr. (2013) 1.

28. K. Ishigami, T. Tajima, A. Nishie et al., Eur. J. Radiol. 74 (2010) e95.

29. B. Viggiano, S. Rose and T. P. Szczykutowicz, Med. Phys. 48 (2021) 1117. 